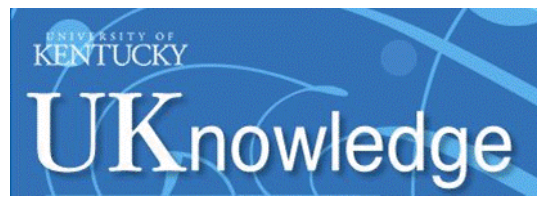

University of Kentucky

UKnowledge

\title{
Design Optimization of Electric Machines with 3D FEA and a New Hybrid DOE-DE Numerical Algorithm
}

\author{
Narges Taran \\ University of Kentucky, narges.taran@uky.edu \\ Vandana Rallabandi \\ University of Kentucky, vandana.rallabandi@uky.edu \\ Dan M. Ionel \\ University of Kentucky, dan.ionel@uky.edu \\ Greg Heins \\ Regal Beloit Corporation, Australia \\ Dean Patterson \\ Regal Beloit Corporation, Australia
}

See next page for additional authors

Follow this and additional works at: https://uknowledge.uky.edu/peik_facpub

Part of the Power and Energy Commons

Right click to open a feedback form in a new tab to let us know how this document benefits you.

\section{Repository Citation}

Taran, Narges; Rallabandi, Vandana; Ionel, Dan M.; Heins, Greg; Patterson, Dean; and Zhou, Ping, "Design Optimization of Electric Machines with 3D FEA and a New Hybrid DOE-DE Numerical Algorithm" (2019). Power and Energy Institute of Kentucky Faculty Publications. 19.

https://uknowledge.uky.edu/peik_facpub/19

This Conference Proceeding is brought to you for free and open access by the Power and Energy Institute of Kentucky at UKnowledge. It has been accepted for inclusion in Power and Energy Institute of Kentucky Faculty Publications by an authorized administrator of UKnowledge. For more information, please contact UKnowledge@lsv.uky.edu. 


\title{
Design Optimization of Electric Machines with 3D FEA and a New Hybrid DOE-DE Numerical Algorithm
}

\author{
Digital Object Identifier (DOI) \\ https://doi.org/10.1109/IEMDC.2019.8785088
}

\section{Notes/Citation Information}

Published in 2019 IEEE International Electric Machines \& Drives Conference (IEMDC).

(C) 2019 IEEE Copyright Notice. "Personal use of this material is permitted. Permission from IEEE must be obtained for all other uses, in any current or future media, including reprinting/republishing this material for advertising or promotional purposes, creating new collective works, for resale or redistribution to servers or lists, or reuse of any copyrighted component of this work in other works."

The document available for download is the authors' manuscript version that is accepted for publication. The final published version is copyrighted by IEEE and will be available as: N. Taran, V. Rallabandi, D. M. Ionel, G. Heins, D.Patterson, and P. Zhou "Design Optimization of Electric Machines with 3D FEA and a New Hybrid DOE-DE Numerical Algorithm," 2019 IEEE International Electric Machines and Drives Conference (IEMDC), San Diego, CA, 2019, pp. 1-6.

Authors

Narges Taran, Vandana Rallabandi, Dan M. Ionel, Greg Heins, Dean Patterson, and Ping Zhou 


\section{Design Optimization of Electric Machines with 3D FEA and a New Hybrid DOE-DE Numerical Algorithm}

\author{
Narges Taran \\ SPARK Lab, ECE Dept. \\ University of Kentucky \\ Lexington, KY, USA \\ narges.taran@uky.edu
}

\author{
Vandana Rallabandi \\ SPARK Lab, ECE Dept. \\ University of Kentucky \\ Lexington, KY, USA \\ vandana.rallabandi@uky.edu
}

\author{
Dan M. Ionel \\ SPARK Lab, ECE Dept. \\ University of Kentucky \\ Lexington, KY, USA \\ dan.ionel@uky.edu
}

\author{
Greg Heins \\ Regal Beloit Corp. \\ Research and Development \\ Rowville, VIC, Australia \\ greg.heins@ regalbeloit.com
}

\author{
Dean Patterson \\ Regal Beloit Corp. \\ Research and Development \\ Rowville, VIC, Australia \\ dean.patterson@regalbeloit.com
}

\author{
Ping Zhou \\ ANSYS, Inc. \\ Research and Development \\ Pittsburgh, PA, USA \\ ping.zhou@ansys.com
}

\begin{abstract}
This paper discusses the multi-objective optimization of axial flux permanent magnet (AFPM) machines with ferrite spoke-type magnets, utilizing 3D finite element models. Three-dimensional finite element analysis is computationally expensive, and furthermore, substantial computation time is expended by optimization algorithms in evaluating low performing designs whose performance is far from the optimum if the search space is not specified correctly. In this regard, this work proposes two new methods for identifying the search space. The search is limited to ranges of input geometric variables where high performing designs are likely to be found. The optimization algorithm utilized is based on surrogate models and differential evolution. It is found that the combined use of these approaches drastically reduces the solution time.
\end{abstract}

Index Terms-Spoke-type, axial flux, optimization, search space, surrogate kriging model, sensitivity analysis.

\section{INTRODUCTION}

Electric machines with three-dimensional (3D) flux path such as axial flux PM (AFPM) machines need to be modeled using computationally expensive 3D finite element analysis (FEA). Other approaches such as quasi-3D, although faster, cannot take 3D flux leakage of spoke-type AFPM machines into account [1]. In order to keep the computation time within affordable limits, the optimization algorithm is required to be particularly efficient with a fewer number of FEA 3D design evaluations. The number of FEA evaluations can be reduced by approaches such as eliminating the insignificant design variables, search space modification, or enhancing the searching capability by directing and ranking the parents [2][4]. Another approach for accelerating the progress of the algorithm is to employ faster function evaluation methods such as computationally efficient FEA [3], [5], or interpolation and surrogate models such as kriging [6], [7]. Although such approaches improve the speed of the optimization process, they still require many design evaluations and are not practical for employing 3D FEA models.

The main focus of this study is to accelerate the 3D FEA based design optimization through a careful definition of the search space. Two new methods for defining the search space are proposed and their performance is compared. One of these methods initially employs a wide search space and iteratively narrows it down. Throughout this paper, this method is referred to as Iterative SES (search space). The other proposed method, makes use of design of experiments (DOE) outcomes to identify a high performing seed reference design. This is unlike most conventional approaches which employ DOE primarily for sensitivity analysis. In the proposed approach the limits of the input variables are specified to be, for example, $\pm 20 \%$ of that of the reference design. Therefore, the search limits are biased by the reference design. Throughout the paper, this method is referred to as Biased SES. The results from the two proposed methods are compared with those from a reference approach, in which the variable limits are defined to be as wide as possible, resulting in a broad search space.

The study is conducted for the spoke-type AFPM machine in Fig. 1. An algorithm based on surrogate models and differential evolution, employing 3D models and elaborated in [8], [9], is utilized for the optimization study. It is demonstrated that the combined use of this surrogate assisted optimization technique, together with the proposed definition of the search space results in substantial savings in computation time.

The paper is structured as follows. The parametric model is introduced in section II. The optimization algorithm is explained in detail in section III. The next section elaborates on the three methods for assigning the search space. The results are discussed and compared in section $\mathrm{V}$ and the study concludes in section VI. 


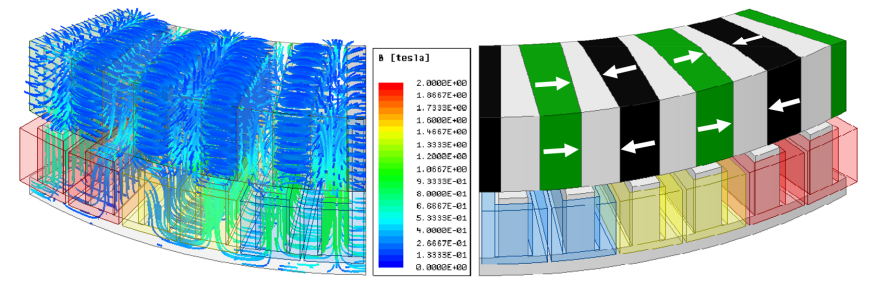

Figure 1: The parametric 3D FEA model for the 40 pole AFPM spoke-type machine with ferrite magnets considered in the optimization study.

\section{Parametric Models and Experimental VALIDATION OF 3D FEA MODELS}

The 40 pole 48 slot spoke-type AFPM machine under study is to be optimally designed with minimum active material cost and electromagnetic loss. The optimization objective functions are defined for the total loss, $F_{l}$, and active material cost, $F_{c}$ :

$$
\begin{gathered}
F_{l}=W_{C u}+W_{c} \\
F_{c}=m_{c}+m_{p m}+3 \cdot m_{C u},
\end{gathered}
$$

where $W_{C u}$, and $W_{c}$ stand for the copper and core losses. PM eddy losses are not significant as ferrite magnets are employed. The total mass of the stator and rotor core are represented with $m_{c}$, and the copper and magnet mass, with $m_{C u}$ and $m_{p m}$, respectively. The mass is calculated in $\mathrm{kg}$ and the steel cost per $\mathrm{kg}$ is considered as the one-unit reference.

This machine employs spoke rectangular ferrite magnets in the rotor, as represented in Fig. 1. Five elite optimization variables, defined as geometric ratios in the 3D FEA model are selected, including: the ratio of stator yoke to total axial length,$k_{s y}$; the ratio of rotor length to total axial length, $k_{r l}$; the ratio of magnet length in magnetization direction to pole pitch in inner diameter, $k_{p m}$; the ratio of slot width to slot pitch in inner diameter, $k_{s w}$; and the split ratio, $\lambda$. Figure 2 and Table I illustrate these variables.

This machine employs tooth concentrated two layer winding. All the studied designs have identical axial length and total outer diameter, including the end coils. Constraining the overall outer diameter results in an additional step in stator width calculation. The coil thickness is considered to be at it's maximum possible, i.e., $\frac{W_{s}}{2}$. Therefore, changes in slot width, enforces the stator diameter, $O D$, to vary as

$$
O D_{t o t}=O D+w_{s} \text {. }
$$

where $O D_{\text {tot }}$ is the constant total outer diameter. On the other hand,

$$
w_{s}=k_{s w} \cdot \tau_{s}=k_{s w} \cdot \frac{\pi \cdot I D}{N_{s}} ; I D=\lambda \cdot O D,
$$

where $N_{s}$ is the number of slots. Solving the system of equations, slot width for constrained overall diameter of $O D_{t o t}$ for the axial flux machine can be obtained as

$$
w_{s}=\frac{O D_{t o t}}{\frac{N_{s}}{k_{s w} \cdot \lambda \cdot \pi}+1}
$$

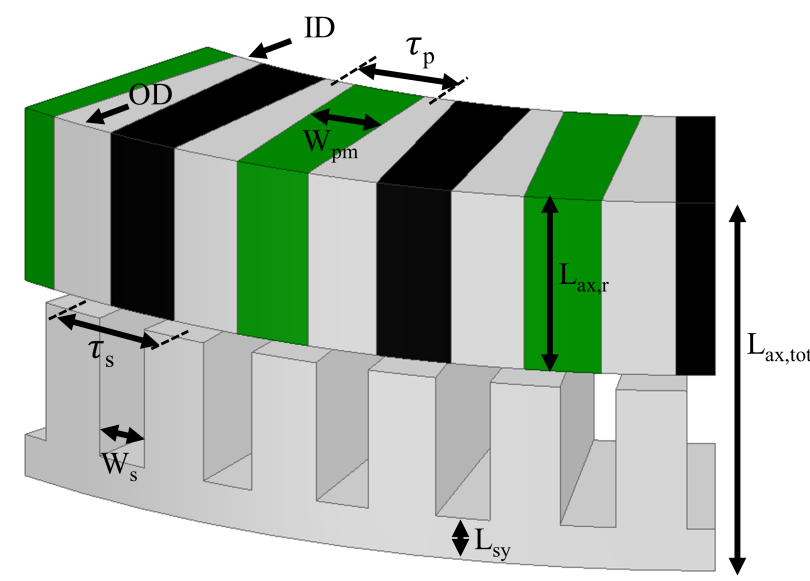

Figure 2: The geometrical variables employed in design optimization.

Table I: The optimization variables and their assigned limits.

\begin{tabular}{llcc}
\hline & & 3 & Search space assignment method \\
Broad and initially & Based SES \\
Variable & Definition & the Iterative SES & Biased \\
\hline$k_{s y}$ & $\frac{L_{s y}}{L_{a x, t o t}}$ & {$[0.11,0.40]$} & {$[0.11,0.18]$} \\
$k_{r l}$ & $\frac{L_{a x, r}}{L_{a x, t o t}}$ & {$[0.20,0.50]$} & {$[0.34,0.47]$} \\
$k_{p m}$ & $\frac{W_{p m}}{\tau_{p}}$ & {$[0.30,0.90]$} & {$[0.55,0.90]$} \\
$k_{s w}$ & $\frac{W_{s}}{\tau}$ & {$[0.45,0.90]$} & {$[0.78,0.90]$} \\
$\lambda$ & $\frac{O D}{I D}$ & {$[0.40,0.85]$} & {$[0.42,0.75]$} \\
\hline
\end{tabular}

The current density is modified from design to design such that all produce the rated torque. The 3D FEA model performed with tetrahedral mesh elements as shown in Fig. 3. The FEA model is validated with experimental measurements of the torque-current characteristics for a prototype ferrite spoke-rotor AFPM machine in Fig. 4.

\section{The surrogate Assisted Multi-objective OPTIMIZATION}

The flowchart for the two level surrogate assisted multiobjective optimization based on differential evolution (DE) algorithm used in this paper, is represented in Fig. 5. This algorithm employs 3D FEA evaluations only for the most promising designs, while the rest are estimated with a local interpolation method known as kriging surrogate model [10], reducing computational time and resources.

The flowchart is composed of an interior level differential evolution that employs kriging models for function evaluations. The kriging surrogate model is a local curve fitting model that, unlike conventional curve fitting methods, does not fit a global polynomial function. The kriging model puts more weight on sampled data points in the vicinity of the unsampled data, providing nonlinear and locally interpolated estimations that are more accurate. The kriging surrogate model can be represented as

$$
\hat{Y}=\hat{X} \beta+r^{T} R^{-1}(Y-X \beta)
$$




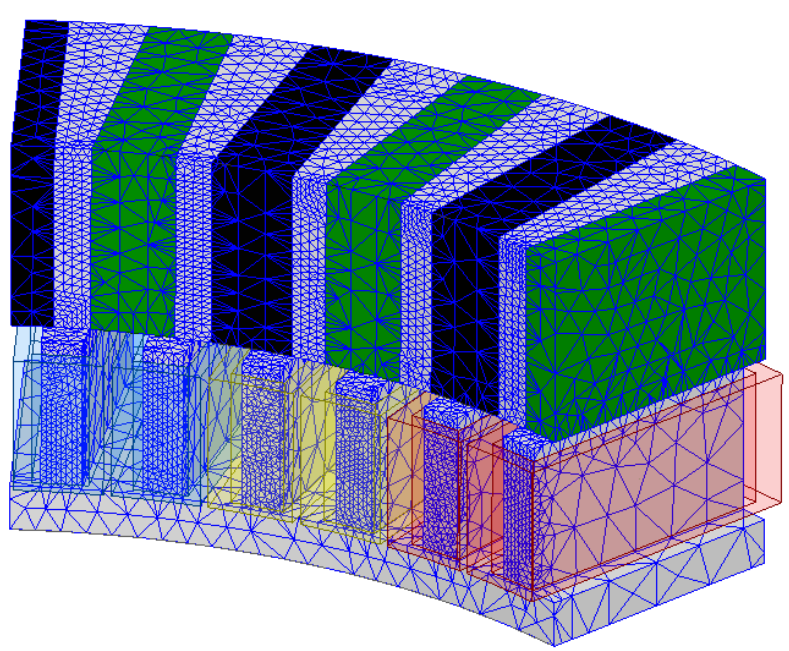

Figure 3: The meshing of the 3D FEA model with tetrahedral elements.

where $\hat{Y}$ is the unsampled design performance to be predicted, based on known sample designs, i.e. $X$ and $Y ; \beta$ is the regression coefficients that can be obtained using methods such as least squares; $r^{T}$ and $R^{-1}$ are derived from a covariance function or semivariogram and a maximum likelihood estimation (MLE) [11]. The first term in (6), known as trend component, is a polynomial function which in case of electric machine optimization problems is usually first order. Higher order trend components may be required for significantly nonlinear problems. The second term in (6), referred to as residual component, takes the spatial correlation among the response values into account.

The two level layout provides an approach to evaluate only the most promising designs with expensive 3D FEAs in the exterior loop, while the interior loop provides an approach for evaluating thousands of designs using inexpensive surrogate interpolations. Details of the algorithm, comparison with conventional methods, and its application for different design problems are provided in [8].

The optimization algorithms with the ranges of design variables as wide as possible, need a large number of function evaluations in order to arrive at the final solution. It is expected that by properly narrowing down the search space the algorithm acquires the Pareto front quicker and this results into an ultra fast method. In this paper two methods of narrowing down the search space are proposed and compared with a reference approach which employs a broader search space. All the studied methods for assigning the optimization search space are included in the flowchart of Fig. 5. The next section elaborates on these techniques of design space assignments.

\section{Reference Design and Search Space SPECIFICATION}

Defining the limits of optimization variables can greatly affect the speed of the optimization algorithm and the final design. If the search space is as wide as possible, a large number of designs would need to be evaluated, making the

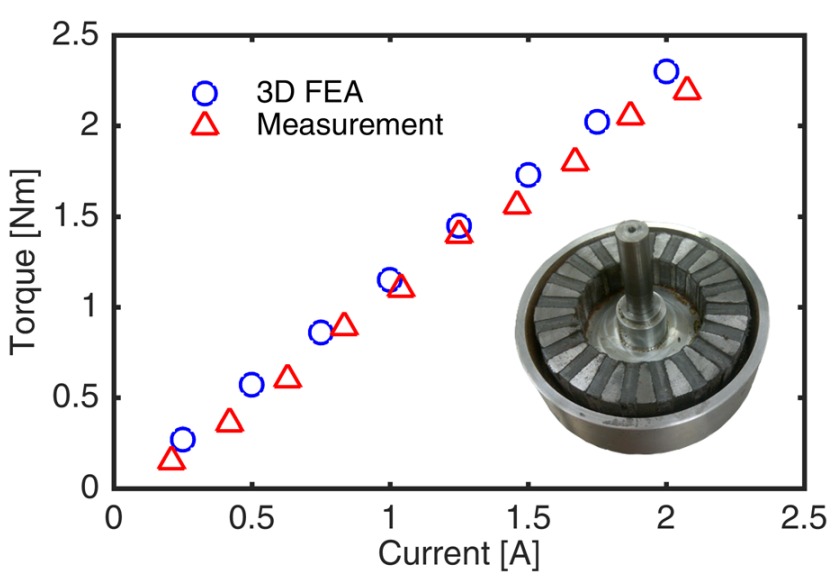

Figure 4: Experimental validation of 3D FEA models.

computation time prohibitively large. On the other hand, with the design limits clustered in a small area, the best trade-off of the objectives may not be achieved. Therefore, the proper specification of the search space is crucial. In this study, three methods for defining the search space are compared.

\section{A. Broad search space specification}

The first method (Broad SES) includes the widest possible ranges for variables' limits. A thorough exploration which may ensure the global optimum result is achieved at the cost of a large number of design evaluations.

\section{B. Iterative search space specification}

The second method (Iterative SES) is proposed to start with similar wide variable ranges and progressively modify them based on the latest Pareto designs. Consequently, the search space shrinks and the speed of the optimization improves. This can be seen as a greedier method and may miss some of the Pareto front designs, compared to the previous approach.

\section{Biased search space specification}

The third method (Biased SES) has narrower ranges for the variables, defining these ranges by taking advantage of DOE outcomes. In contrast with commonly used approaches, the results from the DOE are used here for establishing a reference design and the search space, as explained in the following.

The designs specified by the DOE, conducted over an initially large range, are evaluated using 3D FEA, and in this study, the one with the lowest loss is selected as the reference design. The sensitivity analysis for this design is performed within a specified range, in this case, $\pm 20 \%$ of its variables. As the obtained optimization variable ranges depend on the selected reference design, the search space is biased by its reference. This range is further modified based on sensitivity analysis.

The results of sensitivity analysis for $\pm 20 \%$ range, shown in Fig. 6 with dark blue bars, indicate that reduction in some design variables $\left(k_{s y}\right.$ and $\left.k_{r l}\right)$ and increase in others $\left(k_{p m}\right.$, $k_{s w, i d}$, and $\lambda$ ) decrease the loss. The extended ranges for the variables are defined based on these findings. For instance, 


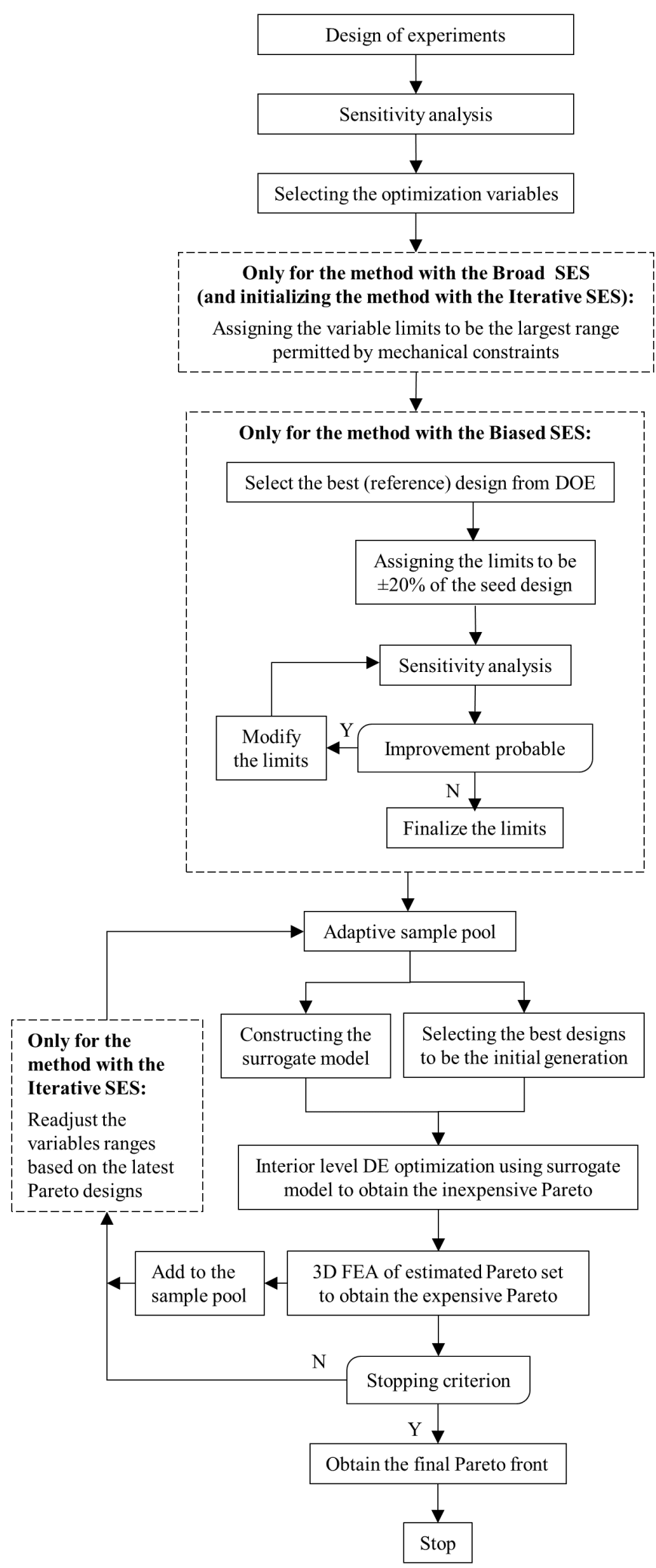

Figure 5: The two-level optimization algorithm with an interior loop based on DE and kriging surrogate models. Three different methods of specifying search space are illustrated. The steps in dashed boxes are specific for different search space assignment approaches.

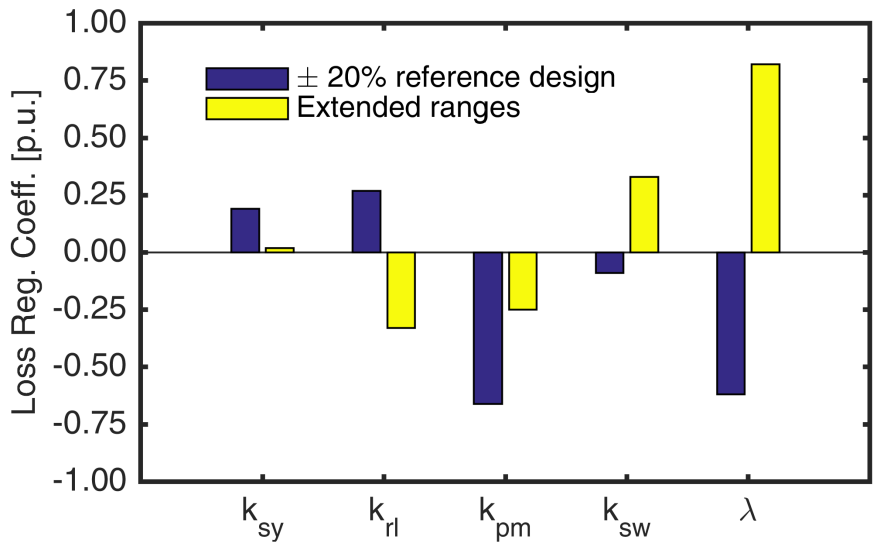

Figure 6: The sensitivity analysis within the range of $\pm 20 \%$ of the reference design variables, and within an extended range to examine the possibility of further reduction in loss.

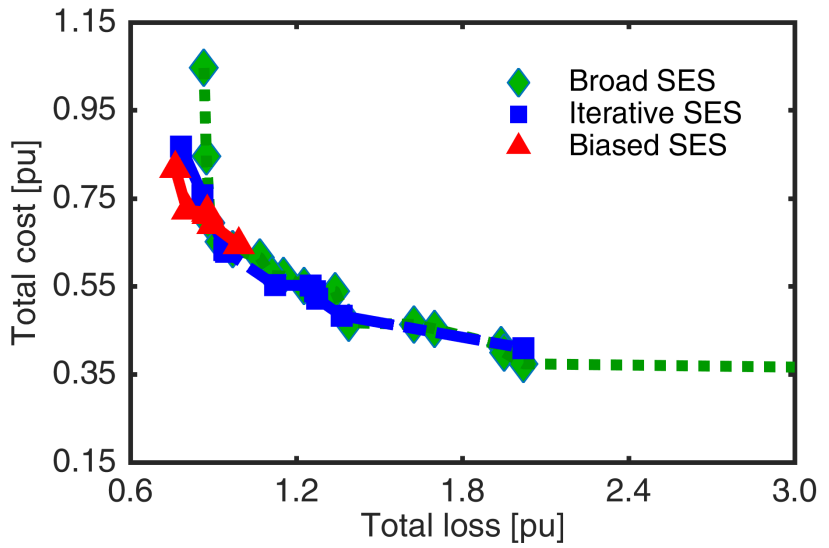

Figure 7: The Pareto front designs obtained from the two-level surrogate assisted optimization with three different methods for search space assignment. The reference design is providing the per-unitization base.

Table II: The number of 3D FEA design evaluations by the surrogate assisted optimization.

\begin{tabular}{l|ccc}
\hline \multirow{2}{*}{$\begin{array}{l}\text { Search space } \\
\text { assignment }\end{array}$} & \multicolumn{3}{|c}{ Number of FEA design evaluations } \\
Initial samples & After initialization & Total \\
\hline Broad & 110 & 70 & 180 \\
Iterative & 110 & 45 & 155 \\
Biased & 70 & 45 & 115 \\
\hline
\end{tabular}

the primary range for $k_{r l}$ is $0.34-0.47$, and sensitivity analysis indicates that lower values result in smaller losses. In order to investigate if further reduction in the value of $k_{r l}$ is beneficial, an extended range is examined within 0.2-0.34. It is observed that the polarity of the regression coefficient changes, as seen in Fig. 6, concluding that further reduction in $k_{r l}$ increases the losses, and hence, the variable is limited between 0.34-0.47. The ranges of other variables to be used for the optimization study are established similarly (Table I). The steps above are exemplified for a spoke-type machine. The consideration of mechanical constraints may also be incorporated in this process. 


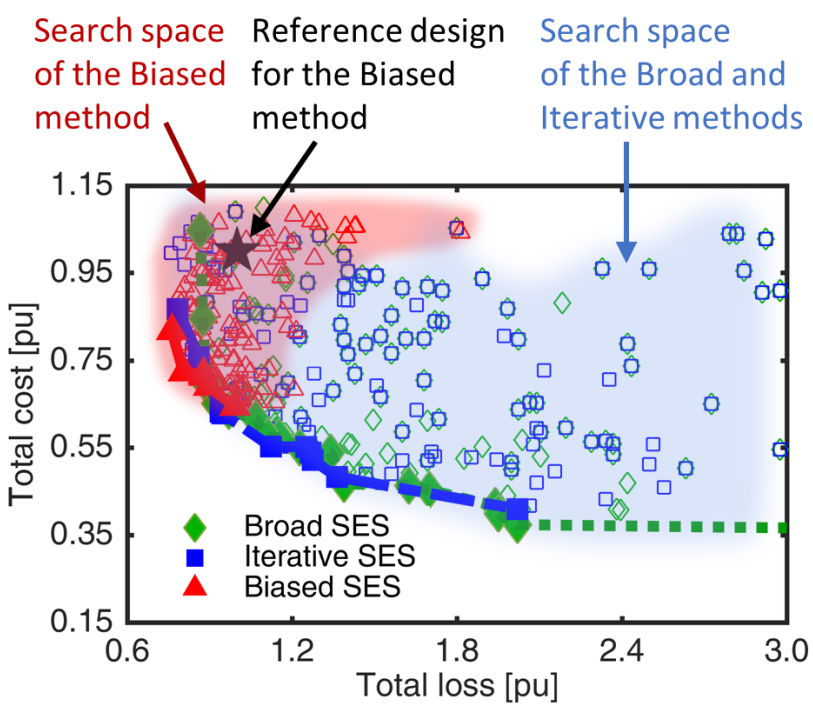

Figure 8: The search space defined with different methods, for the multiobjective optimization of the spoke machine design. All the designs shown in the plot are evaluated with 3D FEA. The reference design is providing the per-unitization base.

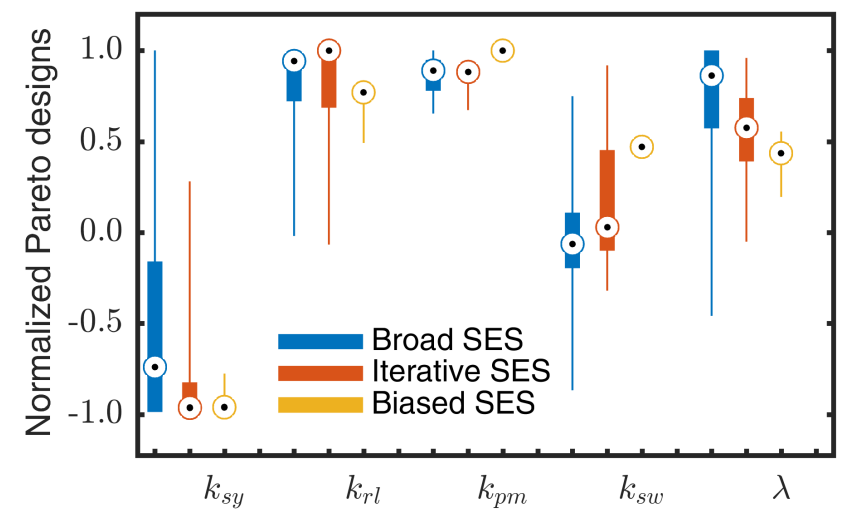

Figure 9: The distribution of variables for the Pareto front designs obtained using different search space assignments.

Table III: The per-unit value of the reference design and a representative optimum design. The total mass, cost, and loss of the reference design represent the per-unitization base.

\begin{tabular}{|c|c|c|c|}
\hline & & Reference & Optimized \\
\hline \multirow{4}{*}{$\stackrel{\tilde{E}}{\tilde{\Sigma}}$} & Steel & 0.72 & 0.35 \\
\hline & Copper & 0.08 & 0.08 \\
\hline & PM & 0.19 & 0.19 \\
\hline & Total & 1.00 & 0.63 \\
\hline \multirow{4}{*}{$\overrightarrow{\tilde{o}}$} & Steel & 0.62 & 0.30 \\
\hline & Copper & 0.22 & 0.21 \\
\hline & PM & 0.17 & 0.16 \\
\hline & Total & 1.00 & 0.68 \\
\hline \multirow{3}{*}{$\begin{array}{l}\tilde{\omega} \\
\stackrel{0}{\Lambda} \\
.\end{array}$} & Steel & 0.17 & 0.14 \\
\hline & Copper & 0.83 & 0.66 \\
\hline & Total & 1.00 & 0.80 \\
\hline \multicolumn{2}{|c|}{ Emag. efficiency [\%] } & 88.7 & 90.6 \\
\hline
\end{tabular}

\section{RESUlts AND Discussion}

The optimization study is conducted with the three discussed methods for defining the search space. The Pareto front is obtained using each of these methods (Fig. 7). The number of 3D FEA design evaluations for each of the methods is given (Table II). It may be noted that the initial design space for the Broad and Iterative methods are identical, hence, both have an equal number of initial samples. The Biased method, having a smaller design space, needs a smaller number of initial FEAs. It is observed that, for the studied optimization problem of the spoke-type AFPM machine, the total number of FEA evaluations is the least when the Biased design space is used. It should be noted that the designs are evaluated using time consuming 3D FEAs and therefore, even the smallest amount of reduction in the number of evaluations is valuable.

The Broad SES assignment, identifies an extended Pareto front and provides various alternative optimum designs to choose between. The absolute limits of the machine design, taking the problem constraints and ratings into account, can be identified using this wide design space, albeit at the cost of larger number of design evaluations.

The Pareto front obtained from proposed Iterative method has fewer designs, nonetheless, it can still provide several optimum options for the designer to select among and it requires fewer FEA runs. The exploitation capability of the optimization algorithm is improved at the cost of the exploration capability (Fig. 7).

The obtained Pareto front highly depends on the location of the search space, which in the third method is biased by the reference design. The design with the least loss from the DOE was selected as the initial reference design for this example, and thus, the search space location is inclined toward the low-loss high-cost zone (Fig. 8). On the other hand, if the reference design was a lower cost and higher loss machine, the search space would be located differently and consequently the Pareto front would yield a different set of optimum designs. Thus, the reference design may be selected based on which of the considered objectives is more important. As a result of the smaller search space, the number of FEA evaluations reduces, as presented in Table II. Due to utilization of a surrogate assisted algorithm, the number of required FEA design evaluation is much smaller than a conventional optimization which usually needs thousands of design evaluations. However, as 3D models are employed, even the smallest amount of reduction in number of analysis saves significant amount of time.

In the example problem, although the emphasis was on obtaining a low loss machine, single-objective optimization was not recommended as cost was also an objective in the second place of priority. In such cases, employing the Biased SES is recommended as a specified location of the Pareto front is of more interest, resulting in fewer 3D FEAs.

Figure 9 represents the distribution of variables for the Pareto front designs. The ranges of the variables for the optimum designs obtained from the Broad SES are the widest, 


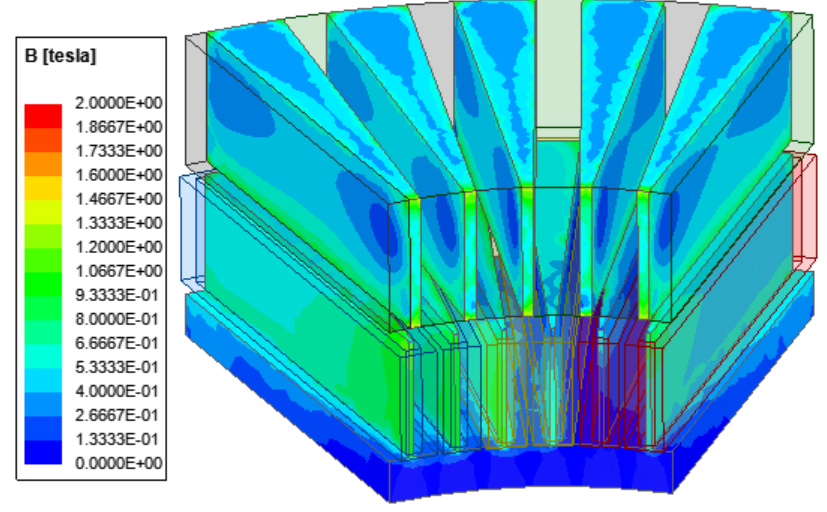

Figure 10: The flux density distribution of the stator and rotor core of the reference design.

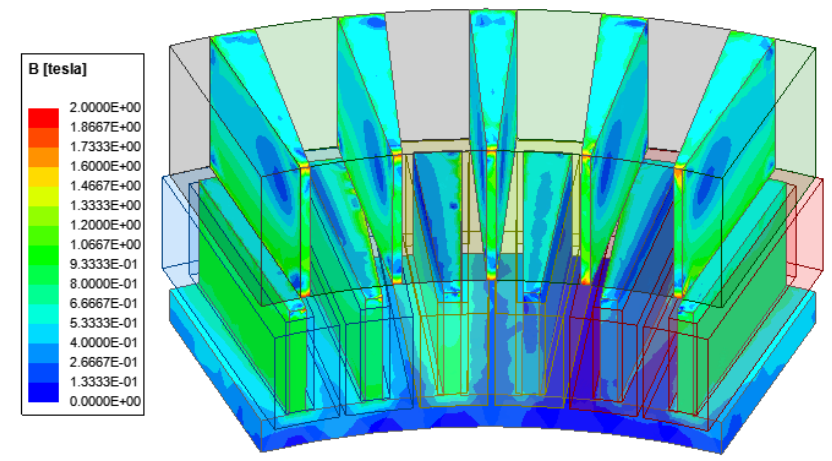

Figure 11: The flux density distribution of the stator and rotor core of the optimized design.

while the Biased SES has tighter ranges. As the designs in the Biased method are inclined toward higher efficiency, it can be deduced that for the studied machine, within the constrained envelope and specified ratings, in order to achieve a higher efficiency, thinner stator yoke and thicker magnets can be used. The rest of the parameters need to be selected through optimization.

The performance of a representative optimum design selected from the Pareto front and the reference design is compared in Table III. The flux density distribution on the rotor and stator core are shown in Fig. 10 and Fig. 11. It should be noted that as the emphasis was more on the loss reduction, the reference design is selected by DOE such that it already has a relatively high efficiency. An additional $2 \%$ increase in the efficiency was achieved through optimization. The optimized design has a larger split ratio with copper and PM mass similar to the reference. Therefore, the slot width and magnet arc ratio have increased to accompany the same copper mass and steel mass with increased inner diameter. As a result of these adjustments the steel mass has significantly reduced, resulting in considerable cost reduction.

\section{CONCLUSION}

The optimized design of a spoke-type AFPM machine was performed with a two-level surrogate assisted algorithm that employs 3D FEA evaluation only for the most promising designs. In addition, the search space is defined through a DOE based method and as a result an order of magnitude reduction in computational time is achieved.

Different methods for the search space definition in multiobjective optimization are proposed and compared. One of these new methods progressively narrows down the variable limits, enhancing the exploitation. The other approach relies on novel applications of the DOE methodology and biases the search space definition based on a reference design and sensitivity analysis. This is especially useful when it is known beforehand which of the objectives is more important. U1timately resulting in a smaller design space, reducing the number of evaluations, thereby greatly improving the speed of the optimization.

\section{ACKNOWLEDGMENT}

The support of Regal Beloit Corporation, University of Kentucky, the L. Stanley Pigman endowment and the SPARK program, and ANSYS Inc. is gratefully acknowledged.

\section{REFERENCES}

[1] S. G. Lee, J. Lee, and W. Kim, "A study on correcting the nonlinearity between stack length and back electromotive force in spoke type ferrite magnet motors," IEEE Transactions on Magnetics, vol. 53, no. 6, pp. 1-4, June 2017.

[2] V. Rallabandi, J. Wu, P. Zhou, D. G. Dorrell, and D. M. Ionel, "Optimal design of a switched reluctance motor with magnetically disconnected rotor modules using a design of experiments differential evolution feabased method," IEEE Transactions on Magnetics, vol. 54, no. 11, pp. 1-5, Nov 2018.

[3] P. Zhang, G. Y. Sizov, D. M. Ionel, and N. A. O. Demerdash, "Establishing the relative merits of interior and spoke-type permanent-magnet machines with ferrite or $\mathrm{NdFeB}$ through systematic design optimization," IEEE Transactions on Industry Applications, vol. 51, no. 4, pp. 29402948, July 2015.

[4] W. Han, C. V. Dang, J. Kim, Y. Kim, and S. Jung, "Global-simplex optimization algorithm applied to fem-based optimal design of electric machine," IEEE Transactions on Magnetics, vol. 53, no. 6, pp. 1-4, June 2017.

[5] G. Y. Sizov, P. Zhang, D. M. Ionel, N. A. O. Demerdash, and M. Rosu, "Automated multi-objective design optimization of PM ac machines using computationally efficient FEA and differential evolution," IEEE Transactions on Industry Applications, vol. 49, no. 5, pp. 2086-2096, Sept 2013.

[6] S. An, S. Yang, and O. A. Mohammed, "A kriging-assisted light beam search method for multi-objective electromagnetic inverse problems," IEEE Transactions on Magnetics, vol. 54, no. 3, pp. 1-4, March 2018.

[7] F. Bittner and I. Hahn, "Kriging-assisted multi-objective particle swarm optimization of permanent magnet synchronous machine for hybrid and electric cars," in 2013 International Electric Machines Drives Conference, May 2013, pp. 15-22.

[8] N. Taran, D. M. Ionel, and D. G. Dorrell, "Two-level surrogate-assisted differential evolution multi-objective optimization of electric machines using 3-D FEA," IEEE Transactions on Magnetics, vol. 54, no. 11, pp. $1-5$, Nov 2018

[9] N. Taran, V. Rallabandi, G. Heins, and D. M. Ionel, "Exploring the efficiency and cost limits of fractional hp axial flux PM machine designs," in 2018 IEEE Energy Conversion Congress and Exposition (ECCE), Sept 2018.

[10] T. Hengl, G. B. Heuvelink, and D. G. Rossiter, "About regressionkriging: From equations to case studies," Computers and Geosciences, vol. 33, no. 10, pp. $1301-1315,2007$.

[11] T. W. Simpson, T. M. Mauery, J. J. Korte, and F. Mistree, "Kriging models for global approximation in simulation-based multidisciplinary design optimization," AIAA Journal, vol. 39, no. 12, pp. 2233-2241, 2001. 